\title{
POWER SERIES FOR INVERSE JACOBIAN ELLIPTIC FUNCTIONS
}

\author{
B. C. CARLSON
}

\begin{abstract}
The 12 inverse Jacobian elliptic functions are expanded in power series by using properties of the symmetric elliptic integral of the first kind. Suitable notation allows three series to include all 12 cases, three of which have been given previously. All coefficients are polynomials in the modulus $k$ that are homogeneous variants of Legendre polynomials. The four series in each of three subsets have the same coefficients in terms of $k$.
\end{abstract}

\section{INTRODUCTION}

Only partial information about power series for inverse Jacobian elliptic functions seems to be available if we ask for series in which all coefficients are algebraic functions of the modulus $k$. If $z=\operatorname{sn}(u, k)$, the inverse function is $u=\operatorname{arcsn}(z, k)$, which was expanded in power series by Kelisky [Ke, (4)]:

$$
\operatorname{arcsn}(z, k)=\sum_{n=0}^{\infty} k^{n} P_{n}\left(\frac{1}{2}\left(k+\frac{1}{k}\right)\right) \frac{z^{2 n+1}}{2 n+1}, \quad|z|<1, \quad 0<k<1,
$$

where $P_{n}$ is the Legendre polynomial of degree $n$. Equation (9) in the same paper gives a similar series for $\operatorname{arcsc}(z, k)$. Lomont and Brillhart [LB, p. 112] cite Kelisky's paper and verify (1.1). Wo shows a series for each of the three functions $\operatorname{arcsp}(z, k)$, $\mathrm{p}=\mathrm{c}$, $\mathrm{d}$, or $\mathrm{n}$, with coefficients containing a ${ }_{2} F_{1}$ polynomial. I have not seen series of the kind specified above for the remaining nine inverse functions. Tölke Tol, (884), (886), (888)] gives the first four terms for each of the six functions included in arcsp and arcps, and three terms (but see the Remarks at the end of this paper) for each of the six functions included in arcqp, where $\mathrm{q}, \mathrm{p} \in\{\mathrm{c}, \mathrm{d}, \mathrm{n}\}, \mathrm{q} \neq \mathrm{p}$. The last two references do not mention Legendre polynomials.

This paper uses known connections between the symmetric elliptic integral $R_{F}$ and the inverse Jacobian functions to derive their infinite power series with "homogeneous Legendre polynomials" as coefficients. We shall show that the coefficients for arcsp, arcps, and two cases of arcqp are the same for a given choice of $\mathrm{p}=\mathrm{c}, \mathrm{d}$, or $\mathrm{n}$. For example, if $\mathrm{p}=\mathrm{n}$, this is true for arcsn, arcns, arccn, and arcdn.

Received by the editor September 6, 2006 and, in revised form, March 1, 2007.

2000 Mathematics Subject Classification. Primary 33E05, 41A58, 33C45; Secondary 33C75.

Key words and phrases. Inverse Jacobian elliptic function, symmetric elliptic integral, Legendre polynomial.

Work at the Ames Laboratory was supported by the Department of Energy-Basic Energy Sciences under Contract No. DE-AC02-07CH11358. 


\section{Derivation of SERIES}

The symmetric incomplete elliptic integral of the first kind, defined by

$$
R_{F}\left(w_{1}, w_{2}, w_{3}\right)=\frac{1}{2} \int_{0}^{\infty} \prod_{j=1}^{3}\left(t+w_{j}\right)^{-1 / 2} d t, \quad\left|\operatorname{ph}\left(w_{j}\right)\right|<\pi
$$

is symmetric and homogeneous of degree $-1 / 2$ in the $w^{\prime}$ s. With $\{\mathrm{p}, \mathrm{q}, \mathrm{r}\}=\{\mathrm{c}, \mathrm{d}, \mathrm{n}\}$ the 12 inverse Jacobian elliptic functions are related to $R_{F}$ by

$$
\begin{aligned}
\operatorname{arcsp}(z, k) & =z R_{F}\left(1+\Delta(\mathrm{q}, \mathrm{p}) z^{2}, 1+\Delta(\mathrm{r}, \mathrm{p}) z^{2}, 1\right) \\
\operatorname{arcps}(z, k) & =z^{-1} R_{F}\left(1+\Delta(\mathrm{q}, \mathrm{p}) z^{-2}, 1+\Delta(\mathrm{r}, \mathrm{p}) z^{-2}, 1\right) \\
\operatorname{arcqp}(z, k) & =\xi R_{F}\left(z^{2}, 1+\Delta(\mathrm{r}, \mathrm{p}) \xi^{2}, 1\right), \quad \xi=\left(\frac{1-z^{2}}{\Delta(\mathrm{p}, \mathrm{q})}\right)^{1 / 2} \\
& =\xi R_{F}\left(1+\Delta(\mathrm{q}, \mathrm{p}) \xi^{2}, 1+\Delta(\mathrm{r}, \mathrm{p}) \xi^{2}, 1\right)
\end{aligned}
$$

where

$$
\Delta(\mathrm{p}, \mathrm{q})=\mathrm{ps}^{2}(u, k)-\mathrm{qs}^{2}(u, k)=-\Delta(\mathrm{q}, \mathrm{p})
$$

and therefore

$$
\Delta(\mathrm{d}, \mathrm{c})=k^{\prime 2}=1-k^{2}, \quad \Delta(\mathrm{n}, \mathrm{d})=k^{2}, \quad \Delta(\mathrm{n}, \mathrm{c})=1 .
$$

In (2.2)-(2.4) the third variable of $R_{F}$ was chosen to be 1 to match (2.16) below. With the help of homogeneity and symmetry, (2.2), (2.3), and (2.4) are easily verified by putting $z=\operatorname{sp}(u, k), z=\operatorname{ps}(u, k)$, and $z=\mathrm{qp}(u, k)$, respectively, to recover in each case the fundamental relation $u=R_{F}\left(\operatorname{ps}^{2}(u, k), \mathrm{qs}^{2}(u, k), \mathrm{rs}^{2}(u, k)\right)$ cdn (1.8)]. Remark 1 at the end of this section may be helpful in the case of (2.4).

Theorem 1. Define

$$
R_{n}(x, y)=R_{n}\left(\frac{1}{2}, \frac{1}{2} ; x, y\right)=\sum_{j=0}^{n} \frac{\left(\frac{1}{2}\right)_{j}\left(\frac{1}{2}\right)_{n-j}}{j !(n-j) !} x^{j} y^{n-j},
$$

where $\left(\frac{1}{2}\right)_{0}=1,\left(\frac{1}{2}\right)_{m}=\frac{1}{2} \frac{3}{2} \frac{5}{2} \cdots\left(m-\frac{1}{2}\right), m=1,2,3, \ldots$ With $\{\mathrm{p}, \mathrm{q}, \mathrm{r}\}=\{\mathrm{c}, \mathrm{d}, \mathrm{n}\}$,

$$
\begin{aligned}
& \operatorname{arcsp}(z, k)=\sum_{n=0}^{\infty} R_{n}(\Delta(\mathrm{p}, \mathrm{q}), \Delta(\mathrm{p}, \mathrm{r})) \frac{z^{2 n+1}}{2 n+1} \\
& \operatorname{arcps}(z, k)=\sum_{n=0}^{\infty} R_{n}(\Delta(\mathrm{p}, \mathrm{q}), \Delta(\mathrm{p}, \mathrm{r})) \frac{(1 / z)^{2 n+1}}{2 n+1} \\
& \operatorname{arcpq}(z, k)=\sum_{n=0}^{\infty} R_{n}(\Delta(\mathrm{p}, \mathrm{q}), \Delta(\mathrm{p}, \mathrm{r})) \frac{\xi^{2 n+1}}{2 n+1}, \quad \xi=\left(\frac{1-z^{2}}{\Delta(\mathrm{p}, \mathrm{q})}\right)^{1 / 2}
\end{aligned}
$$

where all variables may be real or complex. Sufficient conditions for absolute convergence of the series (2.8), (2.9), and (2.10) are given by (2.11), (2.12), and (2.13), respectively:

$$
\begin{aligned}
& \max \{|\Delta(\mathrm{p}, \mathrm{q})|,|\Delta(\mathrm{p}, \mathrm{r})|\}|z|^{2}<1 \\
& \max \{|\Delta(\mathrm{p}, \mathrm{q})|,|\Delta(\mathrm{p}, \mathrm{r})|\}<|z|^{2} \\
& \max \{|\Delta(\mathrm{p}, \mathrm{q})|,|\Delta(\mathrm{p}, \mathrm{r})|\}|\xi|^{2}<1
\end{aligned}
$$


For a proof of the stronger statement that each inequality involves the radius of convergence of the corresponding series, see Remark 2 at the end of this section.

Proof. Since the multivariate hypergeometric $R$-function [Ca, $\S 5.9$ ] includes $R_{F}$ as the special case [Ca, $(9.2-1)]$

$$
R_{F}\left(w_{1}, w_{2}, w_{3}\right)=R_{-1 / 2}\left(\frac{1}{2}, \frac{1}{2}, \frac{1}{2} ; w_{1}, w_{2}, w_{3}\right), \quad\left|\operatorname{ph}\left(w_{j}\right)\right|<\pi,
$$

we can find series expansions of (2.2)-(2.4) from a special case of [Ca, (5.9-4)],

$$
R_{F}\left(1-v_{1}, 1-v_{2}, 1-v_{3}\right)=\sum_{n=0}^{\infty} \frac{\left(\frac{1}{2}\right)_{n}}{n !} R_{n}\left(\frac{1}{2}, \frac{1}{2}, \frac{1}{2} ; v_{1}, v_{2}, v_{3}\right), \quad\left|v_{j}\right|<1
$$

where the polynomial $R_{n}$ [Ca, $\left.\S 6.2\right]$ is symmetric and homogeneous of degree $n$ in the $v$ 's. Putting $v_{3}=0$ and using [Ca, (6.2-6)] with $c_{k}=\frac{3}{2}$ and $c_{p}=\frac{2}{2}$, we have

$$
R_{F}\left(1-v_{1}, 1-v_{2}, 1\right)=\sum_{n=0}^{\infty} \frac{1}{2 n+1} R_{n}\left(\frac{1}{2}, \frac{1}{2} ; v_{1}, v_{2}\right), \quad\left|v_{1}\right|,\left|v_{2}\right|<1,
$$

and (3.9) below shows that the series converges absolutely. In (2.15) and (2.16) the $v$ 's lie in the open unit disk with center 0 in the complex plane. The explicit form of $R_{n}$ with two variables is a special case of [Ca, (6.2-3)] and is reproduced in (2.7) with the parameters $\frac{1}{2}, \frac{1}{2}$ suppressed on the left side for brevity. The relation of (2.7) to the Legendre polynomial $P_{n}$ will be discussed in $\S 3$.

Applying (2.16) to the right side of (2.2)-(2.4), we find

$$
\begin{aligned}
& \operatorname{arcsp}(z, k)=z \sum_{n=0}^{\infty} \frac{1}{2 n+1} R_{n}\left(\Delta(\mathrm{p}, \mathrm{q}) z^{2}, \Delta(\mathrm{p}, \mathrm{r}) z^{2}\right) \\
& \operatorname{arcps}(z, k)=z^{-1} \sum_{n=0}^{\infty} \frac{1}{2 n+1} R_{n}\left(\Delta(\mathrm{p}, \mathrm{q}) z^{-2}, \Delta(\mathrm{p}, \mathrm{r}) z^{-2}\right) \\
& \operatorname{arcqp}(z, k)=\xi \sum_{n=0}^{\infty} \frac{1}{2 n+1} R_{n}\left(\Delta(\mathrm{p}, \mathrm{q}) \xi^{2}, \Delta(\mathrm{p}, \mathrm{r}) \xi^{2}\right), \quad \xi=\left(\frac{1-z^{2}}{\Delta(\mathrm{p}, \mathrm{q})}\right)^{1 / 2}
\end{aligned}
$$

According to (2.16) these series converge absolutely if each variable of $R_{n}$ lies inside the open unit disk, as (2.11)-(2.13) indeed require. Equations (2.8)-(2.10) follow from (2.17)-(2.19) by the homogeneity of $R_{n}$.

Because $\{\mathrm{p}, \mathrm{q}, \mathrm{r}\}=\{\mathrm{c}, \mathrm{d}, \mathrm{n}\}$ the choice of $\mathrm{p}$ determines $\mathrm{q}$ and $\mathrm{r}$ except for their order. For $R_{n}(\Delta(\mathrm{p}, \mathrm{q}), \Delta(\mathrm{p}, \mathrm{r}))$, which is symmetric in its two variables, the order of $\mathrm{q}$ and $\mathrm{r}$ is immaterial, and the coefficients in (2.8)-(2.10) depend only on the choice of $\mathrm{p}=\mathrm{c}, \mathrm{d}$, or $\mathrm{n}$. For each choice there are four inverse functions with the same coefficients: one case of arcsp, one case of arcps, and two cases of arcqp (because q appears also in $\xi)$.

First choosing $\mathrm{p}=\mathrm{n}$, we find from (2.6) that the variables of $R_{n}$ are 1 and $k^{2}$. Then (2.8) becomes

$$
\operatorname{arcsn}(z, k)=\sum_{n=0}^{\infty} R_{n}\left(1, k^{2}\right) \frac{z^{2 n+1}}{2 n+1}, \quad \max \left\{1,\left|k^{2}\right|\right\}|z|^{2}<1 .
$$

Using (2.9) and (2.10) we immediately find the series for arcns, arcen, and arcdn by replacing $z$ on the right-hand side of $(2.20)$ by $1 / z,\left(1-z^{2}\right)^{1 / 2}$, and $\left(\frac{1-z^{2}}{k^{2}}\right)^{1 / 2}$, respectively. 
Choosing $\mathrm{p}=\mathrm{d}$, we find similarly that $(2.8)$ becomes

$$
\operatorname{arcsd}(z, k)=\sum_{n=0}^{\infty} R_{n}\left(1-k^{2},-k^{2}\right) \frac{z^{2 n+1}}{2 n+1}, \quad \max \left\{\left|1-k^{2}\right|,\left|k^{2}\right|\right\}|z|^{2}<1 .
$$

The series for arcds, arccd, and arcnd are found by replacing $z$ on the right-hand side of $(2.21)$ by $1 / z,\left(\frac{1-z^{2}}{1-k^{2}}\right)^{1 / 2}$, and $\left(\frac{z^{2}-1}{k^{2}}\right)^{1 / 2}$, respectively.

Finally, choosing $\mathrm{p}=\mathrm{c}$, we find that $(2.8)$ becomes

$$
\operatorname{arcsc}(z, k)=\sum_{n=0}^{\infty} R_{n}\left(k^{2}-1,-1\right) \frac{z^{2 n+1}}{2 n+1}, \quad \max \left\{\left|1-k^{2}\right|, 1\right\}|z|^{2}<1 .
$$

The series for arccs, arcdc, and arcnc are found by replacing $z$ on the right-hand side of $(2.22)$ by $1 / z,\left(\frac{z^{2}-1}{1-k^{2}}\right)^{1 / 2}$, and $\left(z^{2}-1\right)^{1 / 2}$, respectively.

Remark 1. To understand why the coefficients in (2.8) and (2.9) are the same, note that $u=\operatorname{arcps}(z, k)$ implies $z=\operatorname{ps}(u, k), 1 / z=\operatorname{sp}(u, k)$, and $u=\operatorname{arcsp}(1 / z, k)$. Hence $\operatorname{arcps}(z, k)=\operatorname{arcsp}(1 / z, k)$. A similar argument applies to (2.8) and (2.10) because

$$
\xi^{2}=\frac{1-\mathrm{qp}^{2}}{\Delta(\mathrm{p}, \mathrm{q})}=\frac{1-(\mathrm{qs} / \mathrm{ps})^{2}}{\mathrm{ps}^{2}-\mathrm{qs}^{2}}=1 / \mathrm{ps}^{2}=\mathrm{sp}^{2} .
$$

Thus the quantity with exponent $2 n+1$ in (2.8), (2.9), or (2.10) is in each case sp, which is 0 at $u=0$, as is the sum $u$ of each series.

Remark 2. The right-hand sides of (2.2)-(2.4) have a common form

$$
w R_{F}\left(1-v_{1}, 1-v_{2}, 1\right), \quad\left|\operatorname{ph}\left(1-v_{j}\right)\right|<\pi,
$$

where $w=z, z^{-1}, \xi$ in $(2.2),(2.3),(2.4)$, respectively, and $v_{1}=\Delta(\mathrm{p}, \mathrm{q}) w^{2}, v_{2}=$ $\Delta(\mathrm{p}, \mathrm{r}) w^{2}$. The condition for (2.23), taken from (2.1), states that $1-v_{j}$ lies in the complex plane cut along the nonpositive real axis, and hence $v_{j}$ lies in the complex plane cut along the positive real axis from 1 to $\infty$. In the $w^{2}$-plane this entails a cut rotated away from the positive real axis so that it extends with constant phase from $1 / \Delta(\mathrm{p}, \mathrm{q})$ to $\infty$ and a similar cut from $1 / \Delta(\mathrm{p}, \mathrm{r})$ to $\infty$. Except on these cuts, $(2.23)$ is analytic in $w^{2}$. In particular it is analytic inside the circle with center $w^{2}=0$ that passes through the nearer of the two points $1 / \Delta(\mathrm{p}, \mathrm{q})$ and $1 / \Delta(\mathrm{p}, \mathrm{r})$, one or both of which may be complex (see (2.6)). That circle is the circle of convergence of the power series (2.8)-(2.10) in $w^{2}$ obtained from (2.17)-(2.19) by using the homogeneity of $R_{n}$. The radius of the circle is the radius of convergence

$$
\rho=\min \{|1 / \Delta(\mathrm{p}, \mathrm{q})|,|1 / \Delta(\mathrm{p}, \mathrm{r})|\}=\frac{1}{\max \{|\Delta(\mathrm{p}, \mathrm{q})|,|\Delta(\mathrm{p}, \mathrm{r})|\}} .
$$

Thus the inequalities (2.11)-(2.13), previously described as sufficient, are now seen to be the best possible since they require only that $\left|w^{2}\right|$ be less than $\rho$.

\section{Homogeneous LegendRe POLYNOMIALS}

The polynomials $R_{n}$ have a generating relation that is a special case of [Ca, $(6.6-1)]$ :

$$
\left(1-t(x+y)+t^{2} x y\right)^{-1 / 2}=(1-t x)^{-1 / 2}(1-t y)^{-1 / 2}=\sum_{n=0}^{\infty} t^{n} R_{n}(x, y) .
$$


In terms of the arithmetic and geometric means

$$
\alpha=\frac{x+y}{2}, \quad \gamma=\sqrt{x y},
$$

the generating function is $\left(1-2 \alpha t+\gamma^{2} t^{2}\right)^{-1 / 2}$, and by putting $t=s / \gamma$ we get

$$
\left(1-2 \frac{\alpha}{\gamma} s+s^{2}\right)^{-1 / 2}=\sum_{n=0}^{\infty} \frac{s^{n}}{\gamma^{n}} R_{n}(x, y) .
$$

Comparison with $\mathrm{Ca},(6.7-3)]$ shows that $R_{n}$ is related to the Legendre polynomial $P_{n}$ by

$$
R_{n}(x, y)=\gamma^{n} P_{n}\left(\frac{\alpha}{\gamma}\right)
$$

and reduces to $P_{n}(\alpha)$ if $x y=1$. This close relationship suggests thinking of $R_{n}$ as a homogeneous Legendre polynomial in the variables $\alpha$ and $\gamma$ (see (3.5) below). An example of (3.4) is seen in arcsn, where (2.8) contains the coefficient $R_{n}(\Delta(\mathrm{n}, \mathrm{c}), \Delta(n, \mathrm{~d}))=R_{n}\left(1, k^{2}\right)$. Then $\alpha=\left(1+k^{2}\right) / 2, \gamma=k$, and the right-hand side of $(3.4)$ is $k^{n} P_{n}\left(\frac{1+k^{2}}{2 k}\right)$, the coefficient in (1.1). (Incidentally, in [Ke, (2)] a function $R_{n}(x)$ is defined that can be shown to be $R_{n}\left(1, x^{2}\right)$ in the notation used here.)

Why, then, use $R_{n}$ instead of $P_{n}$ in Theorem 1 ? If $x$ and $y$ are real with opposite signs, all quantities in (2.7) are real, but $\gamma$ and the variable of $P_{n}$ are pure imaginary. This unnecessary complication occurs, for example, in arcsd if $0<k^{2}<1$; it is dealt with in [Ke, (5),(6)] by introducing a second polynomial $S_{n}$ that differs from $P_{n}$ by changing minus signs to plus signs. The polynomial $R_{n}(x, y)$ suffices for both signs of $x y$, and its symmetry was important in showing that the four series in each of three subsets have the same coefficients.

To evaluate $R_{n}$ as a polynomial in $x$ and $y$, we can use (2.7), but if the coefficients $c_{n j}$ in $P_{n}(\alpha)=\sum_{j=0}^{[n / 2]} c_{n j} \alpha^{n-2 j}$ are at hand, we can use (3.4) to find a more concise expression as a homogeneous Legendre polynomial $h_{n}(\alpha, \gamma)$ such that $h_{n}(\alpha, 1)=$ $P_{n}(\alpha)$ :

$$
R_{n}(x, y)=h_{n}(\alpha, \gamma)=\sum_{j=0}^{[n / 2]} c_{n j} \alpha^{n-2 j} \gamma^{2 j}
$$

Thus we have

$$
\begin{aligned}
R_{0}(x, y)=h_{0}(\alpha, \gamma) & =1 \\
R_{1}(x, y)=h_{1}(\alpha, \gamma) & =\alpha=\frac{1}{2}(x+y), \\
R_{2}(x, y)=h_{2}(\alpha, \gamma) & =\frac{1}{2}\left(3 \alpha^{2}-\gamma^{2}\right)=\frac{1}{8}\left(3 x^{2}+2 x y+3 y^{2}\right), \\
R_{3}(x, y)=h_{3}(\alpha, \gamma) & =\frac{1}{2}\left(5 \alpha^{3}-3 \alpha \gamma^{2}\right)=\frac{1}{16}\left(5 x^{3}+3 x^{2} y+3 x y^{2}+5 y^{3}\right), \\
R_{4}(x, y)=h_{4}(\alpha, \gamma) & =\frac{1}{8}\left(35 \alpha^{4}-30 \alpha^{2} \gamma^{2}+3 \gamma^{4}\right) \\
& =\frac{1}{128}\left(35 x^{4}+20 x^{3} y+18 x^{2} y^{2}+20 x y^{3}+35 y^{4}\right) .
\end{aligned}
$$

A recurrence relation that is a special case of [Ca, (5.9-25)] may be more useful for automatic computation,

$$
(n+1) h_{n+1}=(2 n+1) \alpha h_{n}-n \gamma^{2} h_{n-1},
$$


showing by induction that $h_{n}$ is divisible by $\alpha$ for odd $n$.

Note the special cases

$$
R_{n}(x, 0)=\frac{\left(\frac{1}{2}\right)_{n}}{n !} x^{n}, \quad R_{n}(x, x)=x^{n} .
$$

The first equation is obvious from (2.7). The second follows from the nature of $R_{n}$ as a Dirichlet average of $x^{n}$ [Ca, (6.1-1)] or alternatively from a special case of Vandermonde's theorem [Ca, (2.3-1)], which also serves to establish the bound

$$
\left|R_{n}(x, y)\right| \leq[\max \{|x|,|y|\}]^{n} .
$$

This bound provides an elementary route to (2.11)-(2.13) instead of using the convergence condition for $\mathrm{Ca},(5.9-4)]$.

The two parts of (3.8) serve to check the reductions of (2.20)-(2.22) to power series for inverse circular functions if $k=0$ or inverse hyperbolic functions if $k=1$ [AS, 16.6, 4.4, 4.6].

\section{LEADING TERMS}

The coefficients for the leading terms of (2.20)-(2.22) can be shown as explicit polynomials in $k^{2}$ by using (3.6) or (3.7):

$$
\begin{aligned}
\operatorname{arcsn}(z, k)= & z+\frac{1}{6}\left(1+k^{2}\right) z^{3}+\frac{1}{40}\left(3+2 k^{2}+3 k^{4}\right) z^{5} \\
& +\frac{1}{112}\left(5+3 k^{2}+3 k^{4}+5 k^{6}\right) z^{7}+\ldots \\
\operatorname{arcsd}(z, k)= & z+\frac{1}{6}\left(1-2 k^{2}\right) z^{3}+\frac{1}{40}\left(3-8 k^{2}+8 k^{4}\right) z^{5} \\
& +\frac{1}{112}\left(5-18 k^{2}+24 k^{4}-16 k^{6}\right) z^{7}+\ldots \\
\operatorname{arcsc}(z, k)= & z+\frac{1}{6}\left(k^{2}-2\right) z^{3}+\frac{1}{40}\left(3 k^{4}-8 k^{2}+8\right) z^{5} \\
& +\frac{1}{112}\left(5 k^{6}-18 k^{4}+24 k^{2}-16\right) z^{7}+\ldots
\end{aligned}
$$

Corresponding terms for the other nine functions are obtained by replacing $z$ as prescribed after each of (2.20)-(2.22).

Remarks. Recurrence relations equivalent to the special case of (3.7) with $\alpha=$ $\left(1+k^{2}\right) / 2$ and $\gamma=k$ were used by [Wr, (1.4), Table I] and [LB, (8.4), Table 8.1] to list $R_{n}\left(k^{2}, 1\right)$ for $0 \leq n \leq 7$ and $0 \leq n \leq 9$, respectively. [LB] shows the connection with arcsn and the divisibility mentioned after (3.7).

The leading terms given by [Tol, (888)] for the six cases of arcqp were mentioned in the Introduction. They involve powers of $(1-z)^{1 / 2}$ (in present notation) instead of $\left(1-z^{2}\right)^{1 / 2}$. They are obtained from the leading terms of $1-z=1-\mathrm{qp}(u, k)$ as power series in $u^{2}$ [Tol, (816)-(818)]. Reversion of series to get the leading terms of $u^{2}$ as power series in $1-z$ is followed by taking the square root to get leading terms of $u=\operatorname{arcqp}(z, k)$ as series of odd powers of $(1-z)^{1 / 2}$. Each of these two operations would make it increasingly difficult to get higher terms of the final series even if higher terms of the initial series for $1-z$ were readily available.

The equations for arccn given by $[\mathrm{Ke},(6)]$ and $[\mathrm{Wo}$ follow from the power series for $\operatorname{arcsd}$ and the relation $\operatorname{arccn}(z, k)=K(k)-\operatorname{arcsd}\left(z / k^{\prime}\right)$ [La, (3.2.18)].

\section{ACKNOWLEDGMENT}

I thank the referee for asking a question that led to Remark 2 at the end of Section 2 . 


\section{REFERENCES}

[AS] M. Abramowitz and I. A. Stegun, eds., Handbook of Mathematical Functions. US Government Printing Office, Washington, DC, 1964. Reprinted by Dover, New York, 1965. MR0167642 (29:4914)

[Ca] B. C. Carlson, Special Functions of Applied Mathematics, Academic Press, New York, 1977. MR0590943 (58:28707)

[cdn] B. C. Carlson, Symmetry in c, d, n of Jacobian elliptic functions, J. Math. Anal. Appl. 299, 242-253, 2004. MR2091285 (2005h:33044)

[Ke] R. P. Kelisky, Inverse elliptic functions and Legendre polynomials, Amer. Math. Monthly 66, No. 6, 480-483, 1959. MR0103993 (21:2755)

[La] D. F. Lawden, Elliptic Functions and Applications, Springer-Verlag, New York, 1989. MR.1007595 (90h:33001)

[LB] J. S. Lomont and J. Brillhart, Elliptic Polynomials, Chapman \& Hall/CRC, Boca Raton, FL, 2001. MR1887643 (2003h:33001)

[Tol] F. Tölke, Praktische Funktionenlehre, vol. 3, Springer-Verlag, Berlin-New York, 1967. MR $36: 433$

[Wo] http://functions.wolfram.com/EllipticFunctions/

[Wr] S. Wrigge, Calculation of the Taylor series expansion coefficients of the Jacobian elliptic function $\operatorname{sn}(x, k)$, Math. Comp 36, No. 154, 555-564, 1981. MR0606513 (82d:65023)

Ames Laboratory and Department of Mathematics, Iowa State University, Ames, IOWA 50011-3020

E-mail address: bcarlson@scl.ameslab.gov 